\title{
FINITE EXTINCTION AND CONTROL IN SOME DELAY MODELS
}

\author{
Alfonso C. Casal and José M. Vegas
}

Abstract. For a controllable linear time-invariant system $x^{\prime}=A x+b u(t)$ in $\mathbb{R}^{n}$ a general delayed feedback action $u(t)=-k(t) u(t-\tau)$ is proposed so that the solutions of the closed-loop system $x^{\prime}=A x-b k(t) x(t-\tau)$ are driven to zero in finite time. Optimality with respect to some integral performance indices is also analyzed.

Mathematics subject classification (2010): 35R10, 35R35, 35K20.

Keywords and phrases: finite extinction time, delayed feedback control.

\section{REFERENCES}

[1] A. C. CASAL, J. I. DíAZ, On the principle of pseudo-linearized stability: application to some delayed parabolic equations, Nonlinear Analysis, 63 (2005), e997-e1007.

[2] A. C. CASAL, J. I. DíAZ, On the complex Ginzburg-Landau equation with a delayed feedback, Math. Mod. Meth. App. Sci., 16 (2006), 1-17.

[3] A. C. CASAl, J. I. DíAZ, M. Stich, On some delayed nonlinear parabolic equations modeling CO oxidation, Dyn. Contin. Discret. Impuls. Syst. A, 13 (Supp S) (2006), 413-426.

[4] C. CASAL, J. I. DíAZ, M. STich, Control of turbulence in oscillatory reaction-diffusion systems through a combination of global and local feedback, Physical Review E 76, 036209, (2007), 1-9,

[5] C. CASAL, J. I. Díaz, J. M. Vegas, Finite extinction time via delayed feedback actions, Dynamics of Continuous, Discrete and Impulsive Systems, 14, (S2) (2007), 23-27.

[6] C. CASAL, J. I. DíaZ, J. M. Vegas, Finite extinction and null controllability via delayed feedback non-local actions, Nonlinear Anal., 71, 12 (2009), e2018-e2022.

[7] C. CASAL, J. I. DíAZ, J. M. VEgas, Finite extinction time property for a delayed linear problem on a manifold without boundary, Discrete and Continuous Dynamical Systems, (2011), (to appear)

[8] C. CAS AL, J. I. DíAZ, M. STICH, J. M. Vegas, Hopf bifurcation and bifurcation from constant oscillations to a torus path for delayed CGLE, Pgs. 57-76 in Modern Mathematical Tools and Tecnniques in Capturing Complexity, Pardo, L., Balakrishnan, N., Gil, M. A., Eds., Understanding Complex Systems Series, Springer-Verlag, Berlin, 2011.

[9] R. Courant, D. Hilbert, Methods of Mathematical Physics, vol. I., Interscience Publishers, New York, 1966.

[10] E. Sontag, Mathematical Control Theory. Deterministic Finite-Dimensional Systems, SpringerVerlag, New York, 1998.

[11] V. Zoubov, Théorie de la commande, Mir, Moscou, 1978. 